\title{
PROPERTIES OF POSITIVE SOLUTIONS FOR A NONLOCAL REACTION-DIFFUSION EQUATION WITH NONLOCAL NONLINEAR BOUNDARY CONDITION
}

\author{
Chunlai Mu, Dengming Liu, and Shouming Zhou
}

\begin{abstract}
In this paper, we study the properties of positive solutions for the reaction-diffusion equation $u_{t}=\Delta u+\int_{\Omega} u^{p} d x-k u^{q}$ in $\Omega \times(0, T)$ with nonlocal nonlinear boundary condition $u(x, t)=\int_{\Omega} f(x, y) u^{l}(y, t) d y$ on $\partial \Omega \times(0, T)$ and nonnegative initial data $u_{0}(x)$, where $p, q, k, l>0$. Some conditions for the existence and nonexistence of global positive solutions are given.
\end{abstract}

\section{Introduction}

In this paper, we deal with the existence and nonexistence of positive global solutions for the following nonlocal equation with nonlocal nonlinear boundary condition

$$
\begin{cases}u_{t}=\Delta u+\int_{\Omega} u^{p} d x-k u^{q}, & x \in \Omega, t>0 \\ u(x, t)=\int_{\Omega} f(x, y) u^{l}(y, t) d y, & x \in \partial \Omega, t>0, \\ u(x, 0)=u_{0}(x), & x \in \bar{\Omega},\end{cases}
$$

where $\Omega$ is a bounded domain in $\mathbb{R}^{N}$ for $N \geq 1$ with a smooth boundary $\partial \Omega$, $p, q$ and $l$ are positive parameters, the weight function $f(x, y)$ is nonnegative, continuous and defined for $x \in \partial \Omega, y \in \bar{\Omega}$, while the initial data $u_{0}(x) \in L^{2}(\Omega)$ is a nonnegative function and satisfies the compatibility condition $u_{0}(x)=$ $\int_{\Omega} f(x, y) u_{0}^{l}(y) d y$ for $x \in \partial \Omega$.

Many physical phenomena were formulated into nonlocal mathematical models and studied by many authors (see $[2,3,16,20]$ ). In the last few years, a lot of works have been devoted to the study of properties of solutions to parabolic problems involving nonlocal terms. Especially, Wang and Wang [17] considered

Received April 4, 2009; Revised June 3, 2009.

2000 Mathematics Subject Classification. 35B35, 35K57, 35K60, 35K65.

Key words and phrases. reaction-diffusion equation, global existence, blow-up, nonlocal nonlinear boundary condition.

This work is supported in part by NSF of China (10771226) and in part by Natural Science Foundation Project of CQ CSTC (2007BB0124). 
the following reaction-diffusion equation

$$
u_{t}=d \Delta u+\int_{\Omega} u^{p} d x-k u^{q}
$$

with homogeneous Dirichlet boundary condition and positive initial data. They concluded that the blow-up occurs for large initial data if $p>q \geq 1$, and that all solutions exist globally if $1 \leq p<q$. In case of $p=q$, the issue depends on the comparison between $|\Omega|$ and $k$.

In [15], Soufi et al. studied the heat equation of the form

$$
\begin{cases}u_{t}=\Delta u+|u|^{p}-\frac{1}{|\Omega|} \int_{\Omega}|u|^{p} d x, & x \in \Omega, t>0, \\ \frac{\partial u}{\partial n}=0, & x \in \partial \Omega, t>0, \\ u(x, 0)=u_{0}(x), \int_{\Omega} u_{0}(x) d x=0, & x \in \bar{\Omega}\end{cases}
$$

where $1<p \leq 2$. Using energy method and Gamma-convergence technique, they concluded that all solutions blow up in a finite time if the energy of $u_{0}$ is nonpositive. Recently, Jazar and Kiwan [10] generalized the above result, they showed that the solution of (1.3) blow up in a finite time for all $p>1$ while the initial energy is nonpositive.

On the other hand, parabolic equations with nonlocal boundary conditions are also encountered in other physical applications. For instance, in the study of the heat conduction within linear thermoelastcity, Day [4, 5] investigated a heat equation subject to the following boundary conditions

$$
u(-L, t)=\int_{-L}^{L} f_{1}(x) u(x, t) d x, u(L, t)=\int_{-L}^{L} f_{2}(x) u(x, t) d x .
$$

Friedman [8] generalized Day's result to a general parabolic equation

$$
u_{t}=\Delta u+g(x, u), \quad x \in \Omega, t>0,
$$

which is subjected to the following nonlocal boundary condition

$$
u(x, t)=\int_{\Omega} f(x, y) u(y, t) d y .
$$

He established the global existence of solution and discussed its monotonic decay property, and then proved that $\max _{\bar{\Omega}}|u(x, t)| \leq k e^{-\xi t}$ under some hypotheses on $f(x, y)$ and $g(x, u)$.

In addition, parabolic equations with both nonlocal source and nonlocal boundary condition have been studied as well. Such as, Lin and Liu [12] considered the problem of the form

$$
u_{t}=\Delta u+\int_{\Omega} g(u) d x
$$

which is subjected to boundary condition (1.5). They established local existence, global existence and nonexistence of solutions and discussed the blow-up properties of solutions. Furthermore, they derived the uniform blow-up estimate for some special $g(u)$. 
In particular, Wang et al. [19] studied problem (1.2) with nonlocal boundary condition (1.5). They obtained the conditions for existence and nonexistence of global solution. Moreover, they established the precise estimate of the blow-up rate under some suitable hypotheses.

However, reaction-diffusion equations coupled with nonlocal nonlinear boundary condition, such as $u(x, t)=\int_{\Omega} f(x, y) u^{l}(y, t) d y$, to our knowledge, has not been well studied. Very recently, Gladkov and Kim [9] considered the following semilinear heat equation

$$
\begin{cases}u_{t}=\Delta u+c(x, t) u^{p}, & x \in \Omega, t>0, \\ u(x, t)=\int_{\Omega} f(x, y, t) u^{l}(y, t) d y, & x \in \partial \Omega, t>0, \\ u(x, 0)=u_{0}(x), & x \in \bar{\Omega},\end{cases}
$$

where $p, l>0$. They obtained some criteria for the existence of global solution as well as for the solution to blow up in a finite time.

For other works on nonlocal problems, we refer readers to $[13,14,18]$ and references therein.

Motivated by those of above works, we will get blow-up criteria for problem (1.1) with nonlocal nonlinear boundary, which are not only different from situations with the null Dirichlet boundary condition, but also different from situations with boundary condition (1.5). We will show that the weight function $f(x, y)$ and the nonlinear term $u^{l}(y, t)$ in the boundary condition of (1.1) play substantial roles in determining blow-up or not of solution.

In order to state our results, we introduce some useful symbols. Throughout this paper, we let $\lambda$ and $\varphi(x)$ be the first eigenvalue and the corresponding normalized eigenfunction of the problem

$$
-\Delta \varphi(x)=\lambda \varphi, x \in \Omega ; \quad \varphi(x)=0, x \in \partial \Omega,
$$

then

$$
\lambda>0, \quad \varphi(x)>0 \text { and } \int_{\Omega} \varphi(x) d x=1 .
$$

For convenience, we denote

$$
L=\sup _{\bar{\Omega}} \varphi(x), \quad M_{1}=\inf _{\partial \Omega \times \bar{\Omega}} f(x, y), \quad M_{2}=\sup _{\partial \Omega \times \bar{\Omega}} f(x, y) .
$$

The main results of this paper are stated as follows.

Theorem 1.1. Assume that $p<q$ and $l \leq 1$. Then, the problem (1.1) has global solutions for any $f(x, y)$ and any nonnegative initial data.

Theorem 1.2. Assume that $\max \{p, l\}>q \geq 1$ and $\min \left\{\frac{\lambda M_{1}}{L}, \frac{1}{L}\right\}>k$. Then for any $f(x, y)>0$, the solution of problem (1.1) blows up in a finite time if the initial data $u_{0}(x)$ satisfies $\int_{\Omega} u_{0}(x) \varphi(x) d x>1$.

Remark 1.3. In the special case of $k=0$ in (1.1), our results are still true and consistent with those in [9]. 
Theorem 1.4. Assume that $p=q>1$. Then the problem (1.1) has blow-up solutions in a finite time as well as global solutions. More precisely,

(i) if $u_{0}(x)$ is large enough, then for any $f(x, y) \geq 0$, the solution blows up in a finite time.

(ii) if $l \geq 1$ and $\int_{\Omega} f(x, y) d y<1$, the solution exists globally when $u_{0}(x) \leq$ $\rho \psi(x)$ for some $\rho>0$, where $\psi(x)$ is defined in (5.2).

Remark 1.5. If $p=1$ or $p=q=1, l>1$ and $\int_{\Omega} f(x, y) d y<1$, then there exist positive solutions of (1.1) with sufficiently small initial data, which are globally bounded.

Remark 1.6. If $q=1$ or $p=q=1, l>1$, then the solution of problem (1.1) blows up in a finite time for any $f(x, y)>0$ provided that

$$
\int_{\Omega} u_{0}(x) \varphi(x) d x>\left(\frac{\lambda M_{1}}{(\lambda+k) L}\right)^{-\frac{1}{l-1}} .
$$

Remark 1.7. If $p=q=l=1,|\Omega|>k$ and $\int_{\Omega} f(x, y) d y<1$, it is obvious that the problem has no blow-up solution.

In fact, it is easy to verify that $v(t)=\alpha e^{\beta t}$ is a supersolution of (1.1) if $\alpha \geq \max _{x \in \bar{\Omega}} u_{0}(x)$ and $\beta \geq|\Omega|-k$.

Remark 1.8. When $l=1$ in (1.1), then our results agree with those in [19].

The rest of this paper is organized as follows. In Section 2, we establish the comparison principle for problem (1.1). In Sections 3 and 4, we will give the proofs of Theorems 1.1 and 1.2, respectively. Finally, Theorem 1.4 will be proved in Section 5 .

\section{Comparison principle and local existence}

Let $\Omega_{T}=\Omega \times(0, T), S_{T}=\partial \Omega \times(0, T)$ and $\bar{\Omega}_{T}=\bar{\Omega} \times[0, T)$. We begin with the precise definition of a weak solution of problem (1.1) and comparison principle, which will be used in the sequel.

Definition 2.1. A function $u \in L^{2}\left(0, T ; H^{1}(\Omega)\right)$, with $u^{\prime} \in L^{2}\left(0, T ; H^{-1}(\Omega)\right)$, is a weak solution of the problem (1.1) if and only if $\left.u(x, t)\right|_{t=0}=u_{0}(x)$ for all $x \in \bar{\Omega}$, and the equality

$$
\begin{aligned}
& \iint_{\Omega_{T}} u_{t} \phi d x d t+\iint_{\Omega_{T}} \nabla u \cdot \nabla \phi d x d t+\iint_{S_{T}} u \phi d S d t \\
= & \iint_{\Omega_{T}} \phi\left(\int_{\Omega} u^{p} d x-k u^{q}\right) d x d t+\iint_{S_{T}} \phi\left[\int_{\Omega} f(x, y) u^{l}(y, t) d y\right] d S d t
\end{aligned}
$$

holds for all test function $\phi \in L^{2}\left(0, T ; H^{1}(\Omega)\right)$.

In a natural way the notion of a subsolution for (1.1) is given by: 
Definition 2.2. A function $\underline{u} \in L^{2}\left(0, T ; H^{1}(\Omega)\right)$, with $\underline{u}^{\prime} \in L^{2}\left(0, T ; H^{-1}(\Omega)\right)$, is a weak subsolution of the problem (1.1) if and only if $\left.\underline{u}(x, t)\right|_{t=0} \leq u_{0}(x)$ for all $x \in \bar{\Omega}$, and the inequality

$$
\begin{aligned}
& \iint_{\Omega_{T}} \underline{u}_{t} \phi d x d t+\iint_{\Omega_{T}} \nabla \underline{u} \cdot \nabla \phi d x d t+\iint_{S_{T}} \underline{u} \phi d S d t \\
\leq & \iint_{\Omega_{T}} \phi\left(\int_{\Omega} \underline{u}^{p} d x-k \underline{u}^{q}\right) d x d t+\iint_{S_{T}} \phi\left[\int_{\Omega} f(x, y) \underline{u}^{l}(y, t) d y\right] d S d t
\end{aligned}
$$

holds for all test function $0 \leq \phi \in L^{2}\left(0, T ; H^{1}(\Omega)\right)$.

Similarly, a function $\bar{u}(x, t)$ is a supersolution of (1.1) if the reversed inequalities hold in Definition (2.2). A weak solution of (1.1) is a function which is both a subsolution and a supersolution of (1.1). The following comparison principle plays a crucial role in our later proof.

Proposition 2.3 (Comparison principle). Let $\underline{u}$ and $\bar{u}$ be a positive subsolution and supersolution, respectively, with $\underline{u}(x, 0) \leq \bar{u}(x, 0)$ for $x \in \bar{\Omega}$. Then, $\underline{u} \leq \bar{u}$ in $\bar{\Omega}_{T}$.

Proof. We will modify the method in [1] to prove our result.

Step 1. First assume that $p, q, l \geq 1$. Let us denote

$$
\widehat{M}=\max \left\{\|\underline{u}\|_{L^{\infty}\left(\Omega_{T}\right)},\|\bar{u}\|_{L^{\infty}\left(\Omega_{T}\right)}\right\} .
$$

Then we have

$$
\left\|\underline{u}^{q}-\bar{u}^{q}\right\|_{L^{2}\left(\Omega_{T}\right)} \leq q \widehat{M}^{q-1}\|\underline{u}-\bar{u}\|_{L^{2}\left(\Omega_{T}\right)},
$$

and similarly, we have

$$
\left\|\int_{\Omega}\left(\underline{u}^{p}-\bar{u}^{p}\right) d x\right\|_{L^{2}\left(\Omega_{T}\right)} \leq p|\Omega| \widehat{M}^{p-1}\|\underline{u}-\bar{u}\|_{L^{2}\left(\Omega_{T}\right)} .
$$

Consequently

$$
\begin{aligned}
& \left\|\int_{\Omega}\left(\underline{u}^{p}-\bar{u}^{p}\right) d x-k\left(\underline{u}^{q}-\bar{u}^{q}\right)\right\|_{L^{2}\left(\Omega_{T}\right)} \\
\leq & \left(p|\Omega| \widehat{M}^{p-1}+q k \widehat{M}^{q-1}\right)\|\underline{u}-\bar{u}\|_{L^{2}\left(\Omega_{T}\right)},
\end{aligned}
$$

where $|\Omega|$ denotes the Lebesgue measure of $\Omega$.

Let $\omega(x, t)=\underline{u}(x, t)-\bar{u}(x, t)$ and $\omega^{+}=\max \{\omega, 0\}$. Then

$$
\omega^{+} \in L^{2}\left(0, T ; H^{1}(\Omega)\right),
$$


and since $\omega(x, 0) \leq 0$, it follows that $\omega^{+}(x, 0)=0$. Subtracting the defined inequalities for $\underline{u}$ and $\bar{u}$ from each other we get

$$
\begin{aligned}
& \iint_{\Omega_{T}} \omega_{t} \phi d x d t+\iint_{\Omega_{T}} \nabla \omega \cdot \nabla \phi d x d t+\iint_{S_{T}} \omega \phi d S d t \\
\leq & \iint_{\Omega_{T}} \phi\left[\int_{\Omega}\left(\underline{u}^{p}-\bar{u}^{p}\right) d x-k\left(\underline{u}^{q}-\bar{u}^{q}\right)\right] d x d t \\
& +\iint_{S_{T}} \phi\left[\int_{\Omega} f(x, y)\left(\underline{u}^{l}(y, t)-\bar{u}^{l}(y, t)\right) d y\right] d S d t
\end{aligned}
$$

for all $0 \leq \phi \in L^{2}\left(0, T ; H^{1}(\Omega)\right)$. Thus inequality (2.4) remains true for any subcylinder of the form $\Omega_{\tau}=\Omega \times(0, \tau) \subset \Omega_{T}$ and corresponding lateral boundary $S_{\tau}=\partial \Omega \times(0, \tau) \subset S_{T}$. Taking a special test function $\phi=\omega^{+}$in $(2.4)$ and applying (2.3) to $(2.4)$, we find that

$$
\begin{aligned}
& \frac{1}{2}\left\|\omega^{+}(x, \tau)\right\|_{L^{2}(\Omega)}^{2}+\left\|\nabla \omega^{+}\right\|_{L^{2}\left(\Omega_{\tau}\right)}^{2}+\left\|\omega^{+}\right\|_{L^{2}\left(S_{\tau}\right)}^{2} \\
\leq & \left(p|\Omega| \widehat{M}^{p-1}+q k \widehat{M}^{q-1}\right)\left\|\omega^{+}\right\|_{L^{2}\left(\Omega_{\tau}\right)}^{2} \\
& +\iint_{S_{\tau}} \omega^{+}\left[\int_{\Omega} f(x, y)\left(\underline{u}^{l}(y, t)-\bar{u}^{l}(y, t)\right) d y\right] d S d t .
\end{aligned}
$$

Next, our task is to estimate the second term on the right-side of (2.5). Indeed, we first have

$$
\begin{aligned}
& \int_{\Omega} f(x, y)\left(\underline{u}^{l}(y, t)-\bar{u}^{l}(y, t)\right) d y \\
= & \int_{\Omega_{1}} f(x, y)\left(\underline{u}^{l}(y, t)-\bar{u}^{l}(y, t)\right) d y \\
& +\int_{\Omega_{2}} f(x, y)\left(\underline{u}^{l}(y, t)-\bar{u}^{l}(y, t)\right) d y \\
\leq & l M_{2} \widehat{M}^{l-1} \int_{\Omega} \omega^{+}(y, t) d y \\
\leq & l M_{2}|\Omega|^{\frac{1}{2}} \widehat{M}^{l-1}\left\|\omega^{+}(x, t)\right\|_{L^{2}(\Omega)},
\end{aligned}
$$

where $\Omega_{1}=\{y \in \Omega: \underline{u}(y, t)<\bar{u}(y, t)\}, \Omega_{2}=\{y \in \Omega: \underline{u}(y, t) \geq \bar{u}(y, t)\}$. Then by virtue of Young's inequality, we deduce

$$
\begin{aligned}
& \iint_{S_{T}} \omega^{+}\left[\int_{\Omega} f(x, y)\left(\underline{u}^{l}(y, t)-\bar{u}^{l}(y, t)\right) d y\right] d S d t \\
\leq & l M_{2}|\Omega|^{\frac{1}{2}} \widehat{M}^{l-1} \iint_{S_{\tau}}\left\|\omega^{+}(x, t)\right\|_{L^{2}(\Omega)} \omega^{+} d S d t \\
\leq & l M_{2}|\Omega|^{\frac{1}{2}} \widehat{M}^{l-1} \iint_{S_{\tau}}\left(C(\epsilon)\left\|\omega^{+}(x, t)\right\|_{L^{2}(\Omega)}^{2}+\epsilon\left(\omega^{+}\right)^{2}\right) d S d t \\
\leq & l M_{2}|\Omega|^{\frac{1}{2}} \widehat{M}^{l-1}\left(C(\epsilon)|\partial \Omega|\left\|\omega^{+}\right\|_{L^{2}\left(\Omega_{\tau}\right)}^{2}+\epsilon\left\|\omega^{+}\right\|_{L^{2}\left(S_{\tau}\right)}^{2}\right)
\end{aligned}
$$


for any $\epsilon>0$, where $C(\epsilon)$ denotes some positive constant depending only on $\epsilon$. Moreover, we see from the trace theorem that

$$
\left\|\omega^{+}\right\|_{L^{2}\left(S_{\tau}\right)}^{2} \leq \kappa\left(\left\|\nabla \omega^{+}\right\|_{L^{2}\left(\Omega_{\tau}\right)}^{2}+\left\|\omega^{+}\right\|_{L^{2}\left(\Omega_{\tau}\right)}^{2}\right)
$$

where $\kappa$ is a positive constant. Now, let us choose $\epsilon$ sufficiently small such that

$$
l M_{2}|\Omega|^{\frac{1}{2}} \widehat{M}^{p-1} \kappa \epsilon<1 .
$$

From (2.5)-(2.8), it follows that

$$
\left\|\omega^{+}(x, \tau)\right\|_{L^{2}(\Omega)}^{2} \leq C\left\|\omega^{+}\right\|_{L^{2}\left(\Omega_{\tau}\right)}^{2},
$$

where $C$ is some positive constant. Now, we write

$$
y(\tau)=\left\|\omega^{+}(x, \tau)\right\|_{L^{2}(\Omega)}^{2},
$$

then, (2.9) implies that

$$
y(\tau) \leq C \int_{0}^{\tau} y(t) d t \text { for a.e. } 0 \leq \tau \leq T .
$$

By Gronwall's inequality, we know that $y(\tau)=0$ for any $\tau \in[0, T]$. Thus, $\omega^{+}=0$, this means that $\underline{u} \leq \bar{u}$ in $\bar{\Omega}_{T}$ as desired.

Step 2. Consider now the case that $p, q, l<1$, since $\underline{u}$ and $\bar{u}$ are positive, there exists a constant $\mu>0$ such that $\underline{u} \geq \mu>0, \bar{u} \geq \mu>0$. Therefore, we have the following estimate

$$
\begin{aligned}
& \| \int_{\Omega}\left(\underline{u}^{p}-\bar{u}^{p}\right) d x-k\left(\underline{u}^{q}-\bar{u}^{q}\right) \\
\leq & \left(p|\Omega| \mu_{L^{2}(\Omega)}-1+q k \mu^{q-1}\right)\|\underline{u}-\bar{u}\|_{L^{2}(\Omega)},
\end{aligned}
$$

and

$$
\int_{\Omega} f(x, y)\left(\underline{u}^{l}(y, t)-\bar{u}^{l}(y, t)\right) d y \leq l M_{2}|\Omega|^{\frac{1}{2}} \mu^{l-1}\left\|\omega^{+}(x, t)\right\|_{L^{2}(\Omega)} .
$$

Then, the left arguments are the same as those for the case $p, q, l \geq 1$, so we omit them.

Step 3. If $p<1$, or $q<1$, or $l<1$. According to Steps 1 and 2, we can obtain our conclusion easily. The proof of Proposition 2.3 is complete.

Remark 2.4. In [11], if $\underline{u}$ and $\bar{u}$ are a subsolution and supersolution for the corresponding problem, respectively. When $\int_{\Omega} l f(x, y) \chi^{l-1}(y, t) d y \leq 1$ or $f(x, y) \chi^{l-1}(y, t) \leq C$, where $C$ denotes some positive constant and $\chi$ is an intermediate value between $\underline{u}$ and $\bar{u}$, then $\underline{u}(x, 0) \leq \bar{u}(x, 0)$ implies $\underline{u} \leq \bar{u}$ in $\bar{\Omega}_{T}$. From Proposition 2.3, we know that $\underline{u}(x, 0) \leq \bar{u}(x, 0)$ implies $\underline{u} \leq \bar{u}$ in $\bar{\Omega}_{T}$ and we have no restriction on $f(x, y) \chi^{l-1}(y, t)$ here. 
Local in time existence of classical solutions of the problem (1.1) could be obtained by using Schauder's fixed point theorem, the representation formula and the contraction mapping principle as in [7]. The proof is more or less standard, so is omitted here. From comparison principle, we know that the classical solution is positive when $u_{0}(x)$ is positive. We assume that $u_{0}(x)>0$ in the rest of this paper.

\section{Proof of Theorem 1.1}

Proof of Theorem 1.1. Remember that $\lambda$ and $\varphi$ are the first eigenvalue and the corresponding normalized eigenfunction of $-\Delta$ with homogeneous Dirichlet boundary condition. We choose $\delta$ to satisfy that for some $0<\varepsilon<1$,

$$
M_{2} \int_{\Omega} \frac{1}{\delta \varphi(y)+\varepsilon} d y \leq 1
$$

Let

$$
v(x, t)=\frac{c e^{\gamma t}}{\delta \varphi(x)+\varepsilon},
$$

where

$$
\begin{gathered}
c=\max \left\{\sup _{\bar{\Omega}}\left(u_{0}(x)+1\right)(\delta \varphi+\varepsilon), \sup _{\bar{\Omega}}\left[\frac{(\delta \varphi+\varepsilon)^{q}}{k} \int_{\Omega} \frac{1}{(\delta \varphi+\varepsilon)^{p}} d x\right]^{\frac{1}{q-p}}\right\}, \\
\gamma \geq \lambda+\sup _{\bar{\Omega}} \frac{2 \delta^{2}|\nabla \varphi|^{2}}{(\delta \varphi+\varepsilon)^{2}} .
\end{gathered}
$$

A simple computation shows

$$
\begin{aligned}
& v_{t}-\Delta v-\int_{\Omega} v^{p} d x+k v^{q} \\
= & \gamma v-v\left(\frac{\lambda \delta \varphi}{\delta \varphi+\varepsilon}+\frac{2 \delta^{2}|\nabla \varphi|^{2}}{(\delta \varphi+\varepsilon)^{2}}\right)-\int_{\Omega} \frac{c^{p} e^{p \gamma t}}{(\delta \varphi+\varepsilon)^{p}} d x+\frac{k c^{q} e^{q \gamma t}}{(\delta \varphi+\varepsilon)^{q}} \geq 0, \\
& v(x, 0)=\frac{1}{\delta \varphi+\varepsilon} \geq \frac{\sup _{\Omega}\left(u_{0}(x)+1\right)(\delta \varphi(x)+\varepsilon)}{\delta \varphi+\varepsilon}>u_{0}(x) .
\end{aligned}
$$

On the other hand, noticing that $v(x, t)>1$ and $l<1$, we have on the boundary that

$$
\begin{aligned}
v(x, t) & =\frac{c e^{\gamma t}}{\varepsilon}>c e^{\gamma t} \geq \int_{\Omega} f(x, y) \frac{c e^{\gamma t}}{\delta \varphi(y)+\varepsilon} d y=\int_{\Omega} f(x, y) v(y, t) d y \\
& \geq \int_{\Omega} f(x, y) v^{l}(y, t) d y .
\end{aligned}
$$

Combining now (3.3)-(3.5), we see that $v(x, t)$ is a supersolution of (1.1) and $u(x, t)<v(x, t)$ by comparison principle, then the problem (1.1) has global solutions. The proof of Theorem 1.1 is complete. 


\section{Proof of Theorem 1.2}

Proof of Theorem 1.2. The proof is a variant of the eigenfunction method like the one used in [9]. Let $u(x, t)$ be the solution to (1.1). We define the following auxiliary function

$$
J(t)=\int_{\Omega} \varphi(x) u(x, t) d x .
$$

Taking the derivative of $J(t)$ with respect to $t$, we could obtain

$$
\begin{aligned}
J^{\prime}(t) & =\int_{\Omega} \varphi\left(\Delta u+\int_{\Omega} u^{p} d x-k u^{q}\right) d x \\
& =\int_{\partial \Omega} \frac{\partial u}{\partial \nu} \varphi d S-\int_{\Omega} \nabla \varphi \cdot \nabla u d x+\int_{\Omega} u^{p} d x-k \int_{\Omega} \varphi u^{q} d x \\
& =\int_{\Omega} u \Delta \varphi d x-\int_{\partial \Omega} \frac{\partial \varphi}{\partial \nu} u d S+\int_{\Omega} u^{p} d x-k \int_{\Omega} \varphi u^{q} d x \\
& =-\lambda \int_{\Omega} u \varphi d x-\int_{\partial \Omega} \frac{\partial \varphi}{\partial \nu}\left(\int_{\Omega} f(x, y) u^{l}(y, t) d y\right) d S+\int_{\Omega} u^{p} d x-k \int_{\Omega} \varphi u^{q} d x
\end{aligned}
$$

Applying the equality $\int_{\partial \Omega} \frac{\partial \varphi}{\partial \nu} d S=-\lambda \int_{\Omega} \varphi d x=-\lambda$, we get

$$
J^{\prime}(t) \geq \int_{\Omega}\left(-\lambda u+\frac{1}{L} u^{p}+\frac{\lambda M_{1}}{L} u^{l}-k u^{q}\right) \varphi d x .
$$

Let us first assume that $\max \{l, p\}=l$. From (4.2) and Jensen's inequality, it follows that

$$
J^{\prime}(t) \geq-\lambda J+\frac{\lambda M_{1}}{L} J^{l}-k J^{q} \geq-\lambda J+\left(\frac{\lambda M_{1}}{L}-k\right) J^{l}-k .
$$

Next, we look for solution $J(t)$ to $(4.3)$ with $J(0)>1$ on its interval of existence. Since $\frac{\lambda M_{1}}{L}-k>0$ and the function $f(J)=J^{l}$ is convex, there exists $\eta>1$ such that

$$
\left(\frac{\lambda M_{1}}{L}-k\right) J^{l} \geq 2(\lambda J+k), \forall J \geq \eta
$$

It follows easily that if $J(0)>\eta$, then $J(t)$ is increasing on its interval of existence and

$$
J^{\prime}(t) \geq \frac{1}{2} J^{l}
$$

From the above inequality it follows that

$$
\lim _{t \rightarrow T_{0}^{-}} J(t)=+\infty
$$

where

$$
T_{0}=\frac{2}{(l-1) J^{l-1}(0)} .
$$

Then by assumptions in Theorem 1.2, the solution $u(x, t)$ becomes infinite in a finite time. 
We next consider the case $p>q \geq 1$. Owing to (4.2) and Jensen's inequality, we get

$$
J^{\prime}(t) \geq-\lambda J+\left(\frac{1}{L}-k\right) J^{l}-k
$$

Then, since the remainder of the proof is similar to the proof in the case of $l>q \geq 1$, we omit here. This completes the proof.

\section{Proof of Theorem 1.4}

Proof of Theorem 1.4. Firstly, in order to prove our blow-up result, we consider the following well-known nonlocal reaction-diffusion equation

$$
u_{t}=\Delta u+\int_{\Omega} u^{p} d x-k u^{q}
$$

coupled with zero boundary condition and initial data $u_{0}(x)$. Let $v(x, t)$ be the solution of this equation. It is obvious that $v(x, t)$ is a subsolution of the problem (1.1). It is known to all that $v(x, t)$ blows up in a finite time if $u_{0}(x)$ is large enough (see [17, Theorem 3.3]), by Proposition 2.3, we obtain our blow-up result immediately.

Now, we show there exists global solutions if $l>1$ and $\int_{\Omega} f(x, y) d y<1$.

Let $\psi(x)$ be the unique positive solution of the linear elliptic problem

$$
-\Delta \psi(x)=\sigma>0, x \in \Omega ; \quad \psi(x)=\int_{\Omega} f(x, y) d y, x \in \partial \Omega,
$$

where $\sigma$ is chosen such that $0<\psi(x)<1$ (since $\int_{\Omega} f(x, y) d y<1$, there exists such a positive constant $\sigma$ ).

Let

$$
v(x)=\rho \psi(x),
$$

where

$$
0<\rho \leq \min \left\{1,\left(\frac{\sigma}{\int_{\Omega} \psi^{p}(x) d x-k \psi^{p}(x)}\right)^{\frac{1}{p-1}}\right\} .
$$

Calculating directly, we find that

$$
\begin{aligned}
v_{t}-\Delta v=-\Delta v & =\sigma \rho>\rho^{p}\left(\int_{\Omega} \psi^{p}(x) d x-k \psi^{p}(x)\right) \\
& =\int_{\Omega} v^{p}(x) d x-k v^{p}(x) .
\end{aligned}
$$

For $x \in \partial \Omega$, we have that

$$
v(x)=\rho \int_{\Omega} f(x, y) d y>\int_{\Omega} \rho \psi(y) f(x, y) d y \geq \int_{\Omega} v^{l}(y) f(x, y) d y,
$$

where the conditions $v(x)<1$ and $l \geq 1$ are used.

By Proposition 2.3, it follows that $u(x, t)$ exists globally provided that $u_{0}(x)<\rho \psi(x)$. The proof of Theorem 1.4 is complete. 


\section{References}

[1] S. Carl and V. Lakshmikantham, Generalized quasilinearization method for reactiondiffusion equations under nonlinear and nonlocal flux conditions, J. Math. Anal. Appl. 271 (2002), no. 1, 182-205.

[2] D. E. Carlson, Linear Thermoelasticity, in Encyclopedia of Physis, Springer, Berlin, Germany, 1972.

[3] Z. J. Cui and Z. D. Yang, Roles of weight functions to a nonlinear porous medium equation with nonlocal source and nonlocal boundary condition, J. Math. Anal. Appl. 342 (2008), no. 1, 559-570.

[4] W. A. Day, A decreasing property of solutions of parabolic equations with applications to thermoelasticity, Quart. Appl. Math. 40 (1982/83), no. 4, 468-475.

[5] _ Heat Conduction within Linear Thermoelasticity, Springer Tracts in Natural Philosophy, 30. Springer-Verlag, New York, 1985.

[6] K. Deng, Comparison principle for some nonlocal problems, Quart. Appl. Math. 50 (1992), no. 3, 517-522.

[7] L. C. Evans, Partial Differential Equations, Graduate Studies in Mathematics, 19. American Mathematical Society, Providence, RI, 1998

[8] A. Friedman, Monotonic decay of solutions of parabolic equations with nonlocal boundary conditions, Quart. Appl. Math. 44 (1986), no. 3, 401-407.

[9] A. Gladkov and K. I. Kim, Blow-up of solutions for semilinear heat equation with nonlinear nonlocal boundary condition, J. Math. Anal. Appl. 338 (2008), no. 1, 264-273.

[10] M. Jazar and R. Kiwan, Blow-up of a non-local semilinear parabolic equation with Neumann boundary conditions, Ann. Inst. H. Poincare Anal. Non Lineaire 25 (2008), no. 2, $215-218$.

[11] J. Liang, H. Y. Wang, and T. J. Xiao, On a comparison principle for delay coupled systems with nonlocal and nonlinear boundary conditions, in press.

[12] Z. G. Lin and Y. R. Liu, Uniform blowup profiles for diffusion equations with nonlocal source and nonlocal boundary, Acta Math. Sci. Ser. B Engl. Ed. 24 (2004), no. 3, 443450 .

[13] Q. L. Liu, Y. P. Chen, and C. H. Xie, Blow-up for a degenerate parabolic equation with a nonlocal source, J. Math. Anal. Appl. 285 (2003), no. 2, 487-505.

[14] C. M. Peng and Z. D. Yang, Blow-up for a degenerate parabolic equation with a nonlocal source, Appl. Math. Comput. 201 (2008), no. 1-2, 250-259.

[15] A. El Soufi, M. Jazar, and R. Monneau, A gamma-convergence argument for the blow-up of a non-local semilinear parabolic equation with Neumann boundary conditions, Ann. Inst. H. Poincaré Anal. Non Lineaire 24 (2007), no. 1, 17-39.

[16] P. Souplet, Blow-up in nonlocal reaction-diffusion equations, SIAM J. Math. Anal. 29 (1998), no. 6, 1301-1334.

[17] M. X. Wang and Y. M. Wang, Properties of positive solutions for non-local reactiondiffusion problems, Math. Methods Appl. Sci. 19 (1996), no. 14, 1141-1156.

[18] Y. L. Wang, C. L. Mu, and Z. Y. Xiang, Blowup of solutions to a porous medium equation with nonlocal boundary condition, Appl. Math. Comput. 192 (2007), no. 2, $579-585$.

[19] _ Properties of positive solution for nonlocal reaction-diffusion equation with nonlocal boundary, Bound. Value Probl. 2007 (2007), Art. ID 64579, 12 pp.

[20] H. M. Yin, On a class of parabolic equations with nonlocal boundary conditions, J. Math. Anal. Appl. 294 (2004), no. 2, 712-728. 
Chunlai Mu

College of Mathematics and Statistics

Chongqing University

Chongqing 400044, P. R. China

E-mail address: chunlaimu@yahoo.com.cn

Dengming LiU

College of Mathematics and Statistics

Chongqing University

Chongqing 400044, P. R. China

E-mail address: liudengming08@163.com

SHOUMing ZHOU

College of Mathematics and Statistics

Chongling University

Chongqing 400044, P. R. China

E-mail address: zhoushouming76@163.com 